\title{
Fourier magnetic imaging
}

\author{
T. Verduci, ${ }^{1}$ C. Rufo, ${ }^{1}$ A. Berger, ${ }^{1}$ V. Metlushko, ${ }^{2}$ B. Ilic, ${ }^{3}$ and P. Vavassori ${ }^{1,4, a)}$ \\ ${ }^{1}$ CIC nanoGUNE Consolider, E-20018 Donostia-San Sebastian, Spain \\ ${ }^{2}$ Department of Electrical and Computer Engineering, University of Illinois at Chicago, Chicago, Illinois \\ 60607, USA \\ ${ }^{3}$ Cornell Nanofabrication Facility, Cornell University, Ithaca, New York 14853, USA \\ ${ }^{4}$ IKERBASQUE, Basque Foundation for Science, E-48011 Bilbao, Spain
}

(Received 15 June 2011; accepted 7 August 2011; published online 29 August 2011)

\begin{abstract}
We present a methodology for the extraction of the spatial Fourier components of the magnetization profile for an array of nanosized magnetic objects, from diffracted magneto optic Kerr effect measurements. The field dependent magnetization configuration in the unit cell is retrieved by inverse Fourier transform and compared with the predictions of micromagnetic simulations. The comparison demonstrates that the methodology is a powerful optical tool for lens-less diffraction-limited optical imaging of the collective magnetic behavior of nano-structures. (C) 2011 American Institute of Physics. [doi:10.1063/1.3630049]
\end{abstract}

The magneto-optic Kerr effect (MOKE) is widely used in studying technologically relevant magnetic materials. ${ }^{1}$ The MOKE profits from high surface sensitivity, material and depth sensitivity, ${ }^{2}$ its nondestructive character, and the possibility to measure all components of the magnetization vector. $^{3}$ Real time measurements in the sub-ns range and stroboscopic measurements in the sub-ps range have also been demonstrated. ${ }^{4}$ Although conventional measurements are conducted in reflection, recently MOKE has been extended to diffraction geometry, in order to exploit the interference effects of light for arrays of nano-structures. This technique, called diffracted MOKE (D-MOKE) or BraggMOKE ${ }^{5}$ has been used to study the spatial correlation of magnetization at the nano-scale. ${ }^{6}$

Any one of the three distinct configurations used for the conventional MOKE, polar, longitudinal and transverse, ${ }^{7}$ is suitable for the D-MOKE. ${ }^{8}$ However, since the polar and longitudinal geometries depend on at least two of the 4 Fresnel reflectivity coefficients, $r_{p p}$ and $r_{p s}$ or $r_{s s}$ and $r_{s p}$, while the transverse MOKE (TMOKE) configuration depends only on one, $r_{p p}$, the interpretation of the data is greatly simplified for the latter. The TMOKE is sensitive to the component of magnetization that is perpendicular to the scattering plane $\left(m_{\perp}\right)$. In its simplest implementation, the TMOKE is measured as a magnetic field induced change in reflectivity of p-polarized light. In diffraction, the relative variation of intensity of the $n t h$ diffraction order in the scattering plane, $\Delta i_{n}=\Delta \mathrm{I}^{n} / \mathrm{I}_{\mathrm{o}}^{n}$, can be written as ${ }^{9} \Delta i_{\mathrm{n}}=2\left[\left(\Re\left\{A_{n}\right\} \alpha_{n}\right.\right.$ $\left.\left.-\Im\left\{A_{n}\right\} \beta_{n}\right) \Re\left\{f_{m}^{n}\right\}+\left(\Re\left\{A_{n}\right\} \beta_{n}-\Im\left\{A_{n}\right\} \alpha_{n}\right) \Im\left\{f_{m}^{n}\right\}\right]$.

In this expression $f_{m}^{n}$ is the so called magnetic form factor, ${ }^{9}$ i.e., the spatial Fourier transform of the field dependent distribution $m_{\perp}(r, H)$, corresponding to the reciprocal lattice vector $\mathbf{G}_{n}$ of the $n t h$ diffracted beam, i.e., $f_{m}^{n}=\int_{S} m_{\perp}$ $(r, H) \exp \left\{i \mathbf{G}_{n} \cdot \mathbf{r}\right\} d S$, where the integral is carried out over a unit cell $S$ of the lattice. The magnetic form factor describes in a very transparent way the physics involved: in the $n$-order beam the magnetization dependent electric field scattered from different points of the unit cell interferes with a phase accounted for by $\exp \left\{i \mathbf{G}_{n} \cdot \mathbf{r}\right\}$. The coefficients $A_{n}$ are given

${ }^{\text {a)} E l e c t r o n i c ~ m a i l: ~ p . v a v a s s o r i @ n a n o g u n e . e u . ~}$ by $\left(r_{p p \mathrm{~m}}^{n} / r_{p p \mathrm{o}}^{n}\right) / f_{o}^{n}$, where $f_{\mathrm{o}}^{n}=\int_{S} \exp \left\{i \mathbf{G}_{n} \cdot \mathbf{r}\right\} d S, r_{p p \mathrm{o}}^{n}$ and $r_{p p \mathrm{~m}}^{n}$ are the magnetization independent and dependent parts of the reflectivity $r_{p p}^{n}$ generalized to diffraction beams, and $\alpha_{n}$ and $\beta_{n}$ are $\cos \varphi_{n}$ and $\sin \varphi_{n}$, respectively, with $\varphi_{n}$ $=\operatorname{Arg}\left[f_{o}^{n}\right] . f_{o}^{n}$ (thus $\alpha_{n}$ and $\beta_{n}$ ) are independent of magnetization and can be obtained directly from the average intensity of the diffracted beams or simply calculated if the physical shape of the nanostructures is known.

In this Letter, we describe an advanced implementation of D-MOKE that allows us to extract $\Re\left\{f_{m}^{n}\right\}$ and $\Im\left\{f_{m}^{n}\right\}$ directly from measurements and for different in-plane components of the magnetization vector. The clear strength of our approach is that it does not require the knowledge of either the optical and magneto-optical constants or the expression of $r_{p p}^{n}$ generalized to diffraction.

The first step is to adopt a normal incidence geometry (see Fig. 1), which has two main advantages: (i) by adjusting the incident polarization to be in the scattering plane defined by the selected diffracted beam, one can change the magnetization component being probed, combining thus in-plane vector MOKE with D-MOKE; (ii) for $n \rightarrow-n,\left(r_{p p \mathrm{~m}}^{n} / r_{p p \mathrm{o}}^{n}\right)$ simply changes sign while $f_{\mathrm{o}}^{n} \rightarrow\left[f_{\mathrm{o}}^{n}\right]^{*}$ and $f_{m}^{n} \rightarrow\left[f_{m}^{n}\right]^{*}$. These transformations yield to $A_{-n}=-A_{n} f_{\mathrm{o}}^{n} /\left[f_{\mathrm{o}}^{n}\right]^{*}, \Re\left\{f_{m}^{-n}\right\}=\Re\left\{f_{m}^{n}\right\}$,

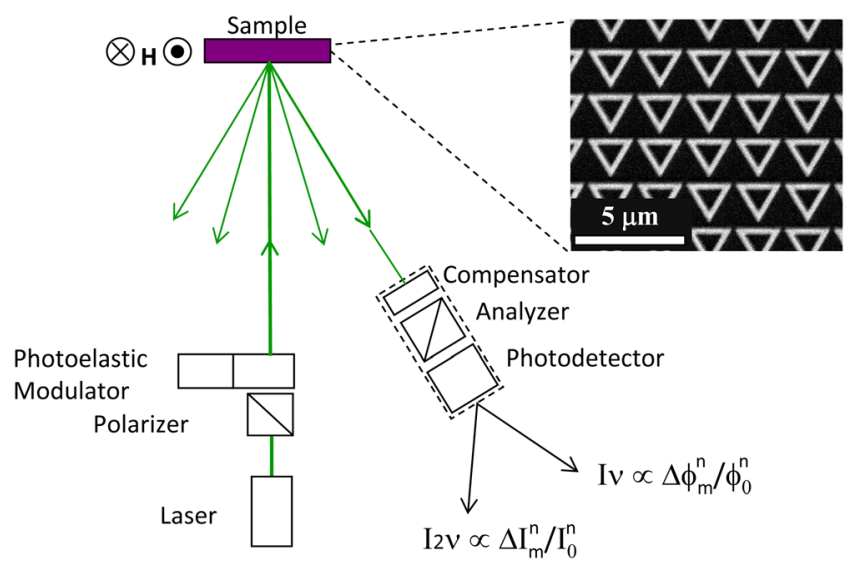

FIG. 1. (Color online) Schematic of the setup implementing null ellipsometry with phase modulation for transverse Kerr effect measurements (see Ref. 12). The inset shows a scanning electron microscopy image of the sample used for the experiments. 


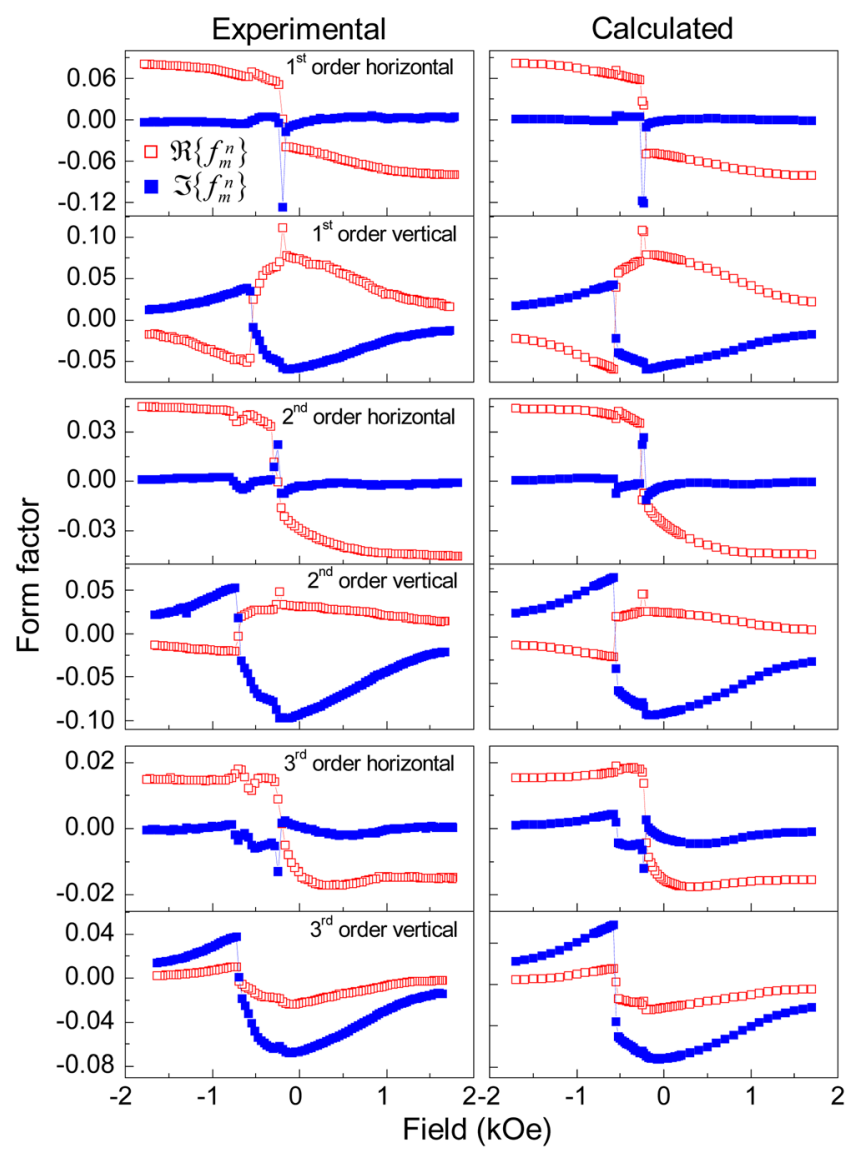

FIG. 2. (Color online) Measured and calculated field dependence of the real $\left(\Re\left\{f_{m}^{n}\right\}\right)$ and immaginary ( $\left.\Im\left\{f_{m}^{n}\right\}\right)$ parts of the magnetic form factors for the array of triangular rings shown in Fig. 1.

and $\Im\left\{f_{m}^{-n}\right\}=-\Im\left\{f_{m}^{n}\right\}$. We finally obtain for $\Delta i_{ \pm n}$ : $\Delta i_{ \pm n}=2\left[\left( \pm \Re\left\{A_{n}\right\} \alpha_{n}-\Im\left\{A_{n}\right\} \beta_{n}\right) \Re\left\{f_{m}^{n}\right\}+\left( \pm \Re\left\{A_{n}\right\} \beta_{n}\right.\right.$ $\left.\left.-\Im\left\{A_{n}\right\} \alpha_{n}\right) \Im\left\{f_{m}^{n}\right\}\right]$.

If the numbers $A_{n}$ were known, the real $\Re\left\{f_{m}^{n}\right\}$ and imaginary $\Im\left\{f_{m}^{n}\right\}$ parts of $f_{m}^{n}$ could be obtained from the measured D-MOKE loops, and the unit cell magnetic configuration $m_{\perp}(r, H)$ reconstructed by inverse Fourier transform. However, the approaches developed to date do not yet allow any reliable calculation of $A_{n}$. The reason for this is that a rigorous theoretical solution to this problem requires a complete vector diffraction approach, including the boundary conditions for all the diffracted waves as well as the magnetooptic contributions to the optical response of the material itself. In the absence of such solutions, the $A_{n}$ have been so far treated as adjustable or fit parameters. Consequently, DMOKE measurements have only been used to confirm proposed magnetic configurations.

In order to overcome these limitations, we exploit the fact that TMOKE involves also a magnetic induced phase change $\Phi$ of reflected light that can be measured by adding a $\lambda / 4$ retarder and a polarizer in front of the photodiode. ${ }^{10}$

The measured variation $\Delta \phi_{ \pm n}=\Delta \Phi^{ \pm n} / \Phi_{\mathrm{o}}^{ \pm n}$ in the diffracted beams can be shown to be: $\Delta \phi_{ \pm n}=2\left[\left( \pm \Im\left\{A_{n}\right\}\right.\right.$ $\left.\left.\alpha_{n}-\Re\left\{A_{n}\right\} \alpha_{n}\right) \Re\left\{f_{m}^{n}\right\}+\left( \pm \Im\left\{A_{n}\right\} \alpha_{n}+\Re\left\{A_{n}\right\} \beta_{n}\right) \Im\left\{f_{m}^{n}\right\}\right]$.

The joint utilization of positive and negative orders leads to the following equalities: $\left(\Delta i_{n}-\Delta i_{-n}\right) \alpha_{n}+\left(\Delta \phi_{n}\right.$ $\left.+\Delta \phi_{-n}\right) \beta_{n}=4 \Re\left\{A_{n}\right\}\left(\alpha_{n}^{2}-\beta_{n}^{2}\right) \Re\left\{f_{m}^{n}\right\}$ and $\left(\Delta i_{n}-\Delta i_{-n}\right) \beta_{n}$ $+\left(\Delta \phi_{n}+\Delta \phi_{-n}\right) \alpha_{n}=4 \Re\left\{A_{n}\right\}\left(\alpha_{n}^{2}-\beta_{n}^{2}\right) \Im\left\{f_{m}^{n}\right\}$.
At magnetic saturation $\Re\left\{f_{m}^{n}\right\}=\Re\left\{f_{0}^{n}\right\}$ so that $4 \Re\left\{A_{n}\right\}\left(\alpha_{n}^{2}-\beta_{n}^{2}\right)$ is the ratio between $\left(\Delta i_{n}-\Delta i_{-n}\right) \alpha_{n}$ $+\left(\Delta \phi_{n}+\Delta \phi_{-n}\right) \beta_{n}$ at saturation and $\Re\left\{f_{\mathrm{o}}^{n}\right\}$.

The field dependence of $\Re\left\{f_{m}^{n}\right\}$ and $\Im\left\{f_{m}^{n}\right\}$ can now be retrieved:

$$
\begin{aligned}
& \Re\left\{f_{m}^{n}\right\}=\frac{\left(\Delta i_{n}-\Delta i_{-n}\right) \alpha_{n}+\left(\Delta \phi_{n}+\Delta \phi_{-n}\right) \beta_{n}}{\left[\left(\Delta i_{n}-\Delta i_{-n}\right) \alpha_{n}+\left(\Delta \phi_{n}+\Delta \phi_{-n}\right) \beta_{n}\right]_{\text {sat }}} \Re\left\{f_{o}^{n}\right\}, \\
& \Im\left\{f_{m}^{n}\right\}=\frac{\left(\Delta i_{n}-\Delta i_{-n}\right) \beta_{n}+\left(\Delta \phi_{n}+\Delta \phi_{-n}\right) \alpha_{n}}{\left[\left(\Delta i_{n}-\Delta i_{-n}\right) \alpha_{n}+\left(\Delta \phi_{n}+\Delta \phi_{-n}\right) \beta_{n}\right]_{\text {sat }}} \Re\left\{f_{o}^{n}\right\} .
\end{aligned}
$$

For particles with mirror symmetry axis perpendicular to the scattering plane Eqs. (1) become very simple since $f_{\mathrm{o}}^{n}$ is real and $\varphi_{n}=0\left(\alpha_{n}=1\right.$ and $\left.\beta_{n}=0\right)$. To illustrate the methodology, we used an array of $25 \mathrm{~nm}$ thick Permalloy triangular rings deposited on Si substrate using e-beam lithography and liftoff techniques. Each ring has a nominal lateral size of $2 \mu \mathrm{m}$ and width of $250 \mathrm{~nm}$, and they are arranged on a slightly rectangular lattice with periods of $\sim 2.0$ and $\sim 2.16 \mu \mathrm{m}$. A scanning electron microscopy image of the sample is shown in the inset of Fig. 1. We selected this sample since the strong shape anisotropy makes the field dependent magnetization configurations easily predictable by means of micromagnetic simulations for the purpose of side to side comparisons. ${ }^{11}$ For the DMOKE experiments we used a solid state green laser (wavelength of $532 \mathrm{~nm}$ ) with its polarization adjusted to be in the scattering plane defined by the diffracted order being measured. Since the sample and $H$ mutual orientation is kept fixed, as sketched in Fig. 1, by changing the diffraction beam with TMOKE we probe different components of the magnetization vector see (Fig. 3). In order to increase the signal to noise ratio, $\Delta \mathrm{I}^{n} / \mathrm{I}_{\mathrm{o}}{ }^{n}$ and $\Delta \Phi^{n} / \Phi_{\mathrm{o}}{ }^{n}$ signals were measured using a configuration based on null ellipsometry with phase modulation. ${ }^{12}$ As discussed in detail in Ref. 12, the phase of the incident beam is modulated at a frequency $v(50 \mathrm{kHz}$ in our case). The DC component and those at $v$ and $2 v$ of the

$$
H=-200 \mathrm{Oe}
$$

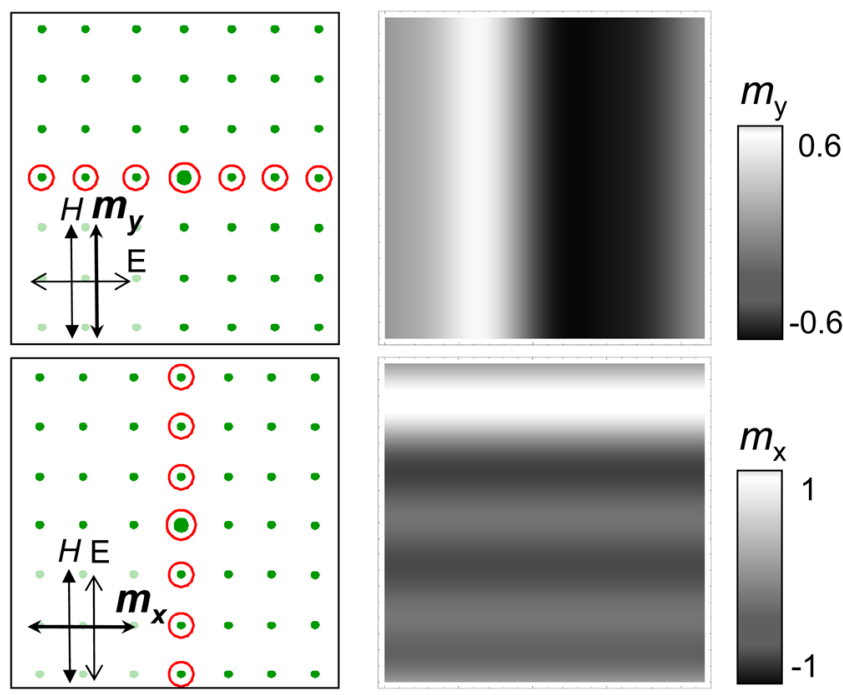

FIG. 3. (Color online) Spatial map of the magnetization profile in the unit cell of the array at $H=-200$ Oe reconstructed via inverse Fourier transform using the form factors of Fig. 2. The left column shows a schematic of the diffraction pattern with the beams used for the reconstruction (marked by circles). 
diffracted beam signals are measured simultaneously using two lock-in amplifiers. By averaging two null measurements with different combinations of compensator and analyzer azimuths, systematic errors, second order effects as well as longitudinal and/or polar MOKE perturbations are eliminated. ${ }^{12}$ The compensator-analyzer-photodiode system is mounted on a specially designed two axis goniometer for fast alignment of the detector to any diffracted beam. The measurement time required to achieve a signal to noise ratio in excess of 50 ranges from 1 to $10 \mathrm{~min}$, depending on the scattered intensity. The complete measurement of the form factors shown in Fig. 2 took about $3 \mathrm{~h}$ with our experimental set-up. Figure 2 shows the field dependence of $\Re\left\{f_{m}^{n}\right\}$ and $\Im\left\{f_{m}^{n}\right\}$ determined using Eqs. (1) up to the 3 rd order in direction perpendicular (horizontal orders, in this case $\varphi_{n}=0$ ) and parallel (vertical orders) to $H$. For a side to side comparison, in Fig. 2 (second column) we show the field dependent $\Re\left\{f_{m}^{n}\right\}$ and $\Im\left\{f_{m}^{n}\right\}$ calculated using $m_{\perp}(r, H)$ computed by micromagnetic simulations. ${ }^{13}$ The agreement between measurements and calculations is remarkable: all the features of calculations are reproduced almost exactly by the experimentally extracted form factors. Using these plots, the map of the magnetization profile at any

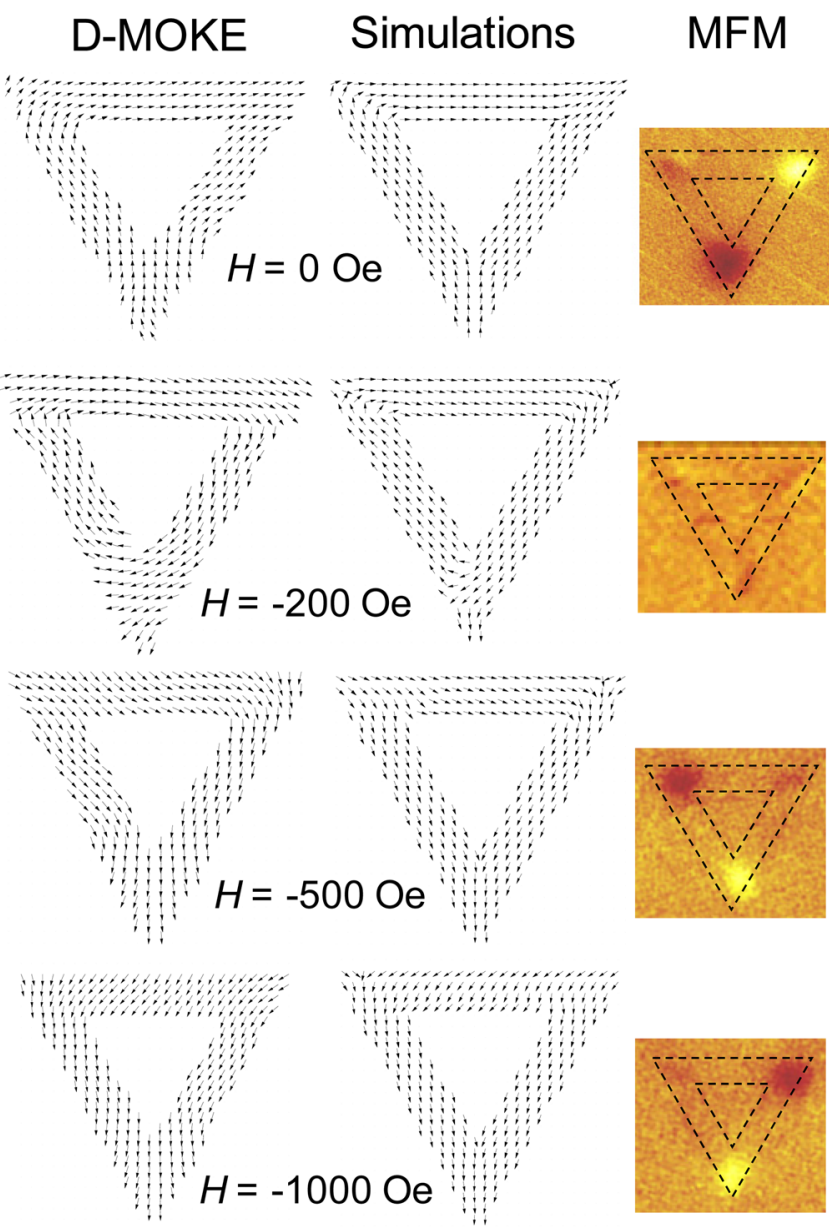

FIG. 4. (Color online) Comparison between experimental and calculated vector maps of the magnetic configuration of a single triangular ring (lateral size of $2 \mu \mathrm{m}$ and width of $250 \mathrm{~nm}$ ) at selected values of the applied field $H$. The experimental maps have been extracted by inverse Fourier transform using the forms factors of Fig. 2. Calculated maps are obtained using micromagnetic simulations. The right-most column shows magnetic force microscopy images taken at zero field after quenching these configurations in a ring through a suitable $H$ history (see Ref. 6).
$H$ can be recovered via inverse Fourier transform. This is illustrated in Fig. 3 using the measured values of $\Re\left\{f_{m}^{n}\right\}$ and $\Im\left\{f_{m}^{n}\right\}$ at $H=-200$ Oe, corresponding to prominent features in the plots in Fig. 2. From these values the spatial maps of $m_{y}(\mathrm{r})$ (horizontal orders) and $m_{x}(\mathrm{r})$ (vertical orders) in the unit cell shown in Fig. 3 are extracted. Noteworthy, to obtain these maps the input of the physical shape of the nanostructures is not needed. This information is used only afterwards to select the portion of the maps in which the nanostructure is located. In Fig. 4 we show the results of the Fourier reconstruction for selected values of $H$ at which particular features are observed in both experimental and calculated plots of Fig. 2, together with the corresponding configurations computed by micromagnetic simulations and magnetic force microscopy images taken at zero field after quenching these states in the rings through a suitable $H$ history. ${ }^{6}$ The agreement between experimental and calculated magnetization distributions is notable and shows that the reversal occurs via a transition between two asymmetric "onion" states passing through a vortex state. ${ }^{11}$ We hereby demonstrated that the D-MOKE methodology presented in this letter allows for the experimental determination of the spatial Fourier components of the field dependent magnetization configuration. This improved implementation of D-MOKE technique provides a powerful, lensless and diffraction-limited, optical tool for the microscopic characterization of the collective magnetic behavior of nanostructures.

We acknowledge funding from the Basque Government under Programs No. PI2009-17 and the Spanish Ministry of Science and Education under Project No. MAT2009-07980. V.M. acknowledges funding from U.S. NSF, Grant No. ECCS-0823813.

${ }^{1}$ Z. Q. Qiu and S. D. Bader, Rev. Sci. Instrum. 71, 1243 (2000).

${ }^{2}$ J. Hamrle, J. Ferré, M. Nyvlt, and S. Vinovsky, Phys. Rev. B 66, 224423 (2002).

${ }^{3}$ A. Berger and M. R. Pufall, Appl. Phys. Lett. 71, 965 (1997); P. Vavassori, ibid. 77, 1605 (2000).

${ }^{4}$ Y. Acremann, C. H. Back, M. Buess, O. Portmann, A. Vaterlaus, D. Pescia, and H. Melchior, Science 290, 492 (2000).

${ }^{5}$ M. Grimsditch and P. Vavassori, J. Phys.: Condens. Matter. 16, R275 (2004).

${ }^{6}$ I. Guedes, M. Grimsditch, V. Metlushko, P. Vavassori, R. Camley, and B. Ilic, Phys. Rev. B 66, 014434 (2002); P. Vavassori, M. Grimsditch, V. Novosad, V. Metlushko, and B. Ilic, ibid. 67, 134429 (2003); P. Vavassori, N. Zaluzec, V. Metlushko, V. Novosad, and M. Grimsditch, ibid. 69, 214404 (2004).

${ }^{7}$ M. Freiser, IEEE Trans. Magn. 4, 152 (1968).

${ }^{8}$ A. Westphalen, K. Theis-Brohl, H. Zabel, K. Rott, and H. Bruckl, J. Magn. Magn. Mater. 302, 181 (2006).

${ }^{9}$ M. Grimsditch, P. Vavassori, V. Novosad, V. Metlushko, H. Shima, Y. Otani, and K. Fukamichi, Phys. Rev. B 65, 172419 (2002).

${ }^{10}$ C. Penfold, R. T. Collins, A. P. B. Tufaile, and Y. Souche, J. Magn. Magn. Mater. 242-245, 964 (2002).

${ }^{11}$ P. Vavassori, O. Donzelli, M. Grimsditch, V. Metlushko, and B. Ilic, J. Appl. Phys. 101, 023902 (2007); P. Vavassori, D. Bisero, V. Bonanni, A. Busato, M. Grimsditch, K. M. Lebecki, V. Metlushko, and B. Ilic, Phys. Rev. B 78, 174403 (2008).

${ }^{12}$ K. Postava, A. Maziewski, A. Stupakewicz, A. Wawro, L. T. Baczewski, S. Vinovsky, and T. Yamaguchi, J. Eur. Opt. Soc. Rapid Publ. 1, 06017 (2006).

${ }^{13}$ Micromagnetic simulations have been performed on a single nanostructure using the OOMMF public domain simulation platform [M. J. Donahue, D. G. Porter, 1999 OOMMF User's Guide, Version 1.0. Interagency Report NISTIR 6376 (Gaithersburg, National Institute of Standards and Technology)] with a cubic cell of $10 \mathrm{~nm}$ side. We verfied in a few cases that the use of a smaller cell of $5 \mathrm{~nm}$ size does not affect the results of simulations. 